\title{
A Family of Variable-Metric Methods Derived by Variational Means
}

\author{
By Donald Goldfarb
}

\begin{abstract}
A new rank-two variable-metric method is derived using Greenstadt's variational approach [Math. Comp., this issue]. Like the Davidon-Fletcher-Powell (DFP) variable-metric method, the new method preserves the positive-definiteness of the approximating matrix. Together with Greenstadt's method, the new method gives rise to a one-parameter family of variable-metric methods that includes the DFP and rank-one methods as special cases. It is equivalent to Broyden's one-parameter family [Math. Comp., v. 21, 1967, pp. 368-381]. Choices for the inverse of the weighting matrix in the variational approach are given that lead to the derivation of the DFP and rank-one methods directly.
\end{abstract}

In the preceding paper [6], Greenstadt derives two variable-metric methods, using a classical variational approach. Specifically, two iterative formulas are developed for updating the matrix $H_{k}$, (i.e., the inverse of the variable metric), where $H_{k}$ is an approximation to the inverse Hessian $G^{-1}\left(x_{k}\right)$ of the function being minimized.*

Using the iteration formula

$$
H_{k+1}=H_{k}+E_{k}
$$

to provide revised estimates to the inverse Hessian at each step, Greenstadt solves for the correction term $E_{k}$ that minimizes the norm

$$
N\left(E_{k}\right)=\operatorname{Tr}\left(W E_{k} W E_{k}{ }^{T}\right)
$$

subject to the conditions

$$
E_{k}^{T}=E_{k}
$$

and

$$
E_{k} y_{k}=\sigma_{k}-H_{k} y_{k} .
$$

$W$ is a positive-definite symmetric matrix and $\operatorname{Tr}$ denotes the trace.

The first condition is a symmetry condition which ensures that all iterates $H_{k}$ will be symmetric as long as the initial estimate $H_{0}$ is chosen to be symmetric. The second condition ensures that the updated matrix $H_{k+1}$ satisfies the equation

$$
H_{k+1} y_{k}=\sigma_{k}
$$

and hence, that the method is of the "quasi-Newton" type [1].

Received June 30, 1969, revised August 4, 1969.

AMS Subject Classifications. Primary 30, Secondary 10.

Key Words and Phrases. Unconstrained optimization, variable-metric, variational methods, Davidon method, rank-one formulas.

* The reader is referred to Greenstadt's paper [6] for a more detailed discussion of variablemetric methods and for definitions of some of the terms used here. 
If the function being minimized were quadratic, $H_{k+1}$ would operate on the vector $y_{k}$ as would the matrix $G^{-1}$. The norm chosen by Greenstadt is essentially a weighted Euclidean norm.

Solving this constrained minimization problem using Lagrange multipliers, Greenstadt obtained the following formula for $E_{k}$ :

$$
\begin{aligned}
E_{k}=\frac{1}{\left(y^{T} M y\right)}\left\{\sigma y^{T} M+M y \sigma^{T}-H y y^{T} M\right. & -M y y^{T} H \\
& \left.-\frac{1}{\left(y^{T} M y\right)}\left[\left(y^{T} \sigma\right)-\left(y^{T} H y\right)\right] M y y^{T} M\right\},
\end{aligned}
$$

where $M=W^{-1}$.

If the current approximation $H$ to $G^{-1}$ is substituted for $M$, Greenstadt's first formula is obtained:

$$
E_{I I}=\frac{1}{\left(y^{T} H y\right)}\left\{\sigma y^{T} H+H y \sigma^{T}-\left[1+\left(\frac{y^{T} \sigma}{y^{T} H y}\right)\right] H y y^{T} H\right\} .
$$

(Throughout the remainder of the note no superscript will indicate the $k$ th iterate and a $\left(^{*}\right)$ superscript will denote the $(k+1)$ st iterate.)

If, instead, $H^{*}$ is substituted for $M$ in Eq. (3),

$$
E_{H^{*}}=\frac{1}{\left(y^{T} \sigma\right)}\left\{-\sigma y^{T} H-H y \sigma^{T}+\left[1+\frac{\left(y^{T} H y\right)}{\left(y^{T} \sigma\right)}\right] \sigma \sigma^{T}\right\}
$$

is obtained. The above two correction terms appear to be similar, at least in part, to both the Davidon-Fletcher-Powell (or DFP) rank-2 correction term

$$
E_{R 2}=\frac{\sigma \sigma^{T}}{\sigma^{T} y}-\frac{H y y^{T} H}{y^{T} H y}
$$

and the rank-1 correction term [1], [3], and [7]

$$
E_{R 1}=\frac{(\sigma-H y)(\sigma-H y)^{T}}{(\sigma-H y)^{T} y} .
$$

In fact, all four corrections terms $E_{H}, E_{I I^{*}}, E_{R 1}$, and $E_{R 2}$ give rise to algorithms that locate the exact minimum of a strictly convex quadratic objective function of $N$ variables in $N$ steps. They also result in a matrix $H$ which after those $N$ steps is exactly equal to $G^{-1}$. Proofs of this property, which we shall refer to as "exactness" following Broyden [1], were given for $E_{R 2}, E_{R 1}$, and $E_{I}$ by Fletcher and Powell [4], Broyden [1], and Bard [6, Appendix], respectively.

It is easy to show that this property also holds for variable-metric algorithms with correction term $E_{H^{*}}$. For example, Bard's proof may be followed almost entirely, except for some obvious and trivial changes.

$E_{R 2}$ and $E_{I^{*}}$, moreover, share the additional property of preserving the positivedefiniteness of the approximating matrix $H$. This ensures the stability of the corresponding variable-metric algorithms that search for a minimum along the direction $-H g$ at each step. Fletcher and Powell proved this for $E_{R 2}$. The proof for $E_{H^{*}}$ follows from the observation that 


$$
x^{T}\left(E_{H^{*}}-E_{R 2}\right) x=x^{T} E_{H}{ }^{*} x-x^{T} E_{R 2} x=\frac{\left[\left(y^{T} H y\right)\left(x^{T} \sigma\right)-\left(y^{T} \sigma\right)\left(x^{T} H y\right)\right]^{2}}{\left(y^{T} \sigma\right)^{2}\left(y^{T} H y\right)} \geqq 0 .
$$

It may seem then that the iteration scheme $H^{*}=H+E_{H^{*}}$ would be less likely to generate a sequence of matrices $\left\{H_{i}\right\}$ that tends toward singularity than would the DFP iteration scheme $H^{*}=H+E_{R 2}$. One should not count this apparent improvement too heavily, for the behavior of a variable-metric algorithm and its convergence to a stationary point depend upon the sequence $\left\{H_{i}\right\}$ being bounded above as well as being bounded away from singularity [5].

The resemblances between the correction terms $E_{R 2}, E_{R 1}, E_{H}$ and $E_{H^{*}}$ suggest that each can be written as a linear combination of the others. This is indeed the case: $E_{R 2}$ and $E_{R 1}$ can be expressed directly as weighted sums of $E_{I I}$ and $E_{H^{*}}$, and vice versa.

where

$$
\begin{aligned}
& E_{R 2}=\frac{\left(y^{T} H y\right) E_{H}+\left(y^{T} \sigma\right) E_{H^{*}}}{y^{T} H y+y^{T} \sigma}=\frac{\left(y^{T} H y\right) E_{H}+\left(y^{T} H^{*} y\right) E_{H^{*}}}{y^{T} H y+y^{T} H^{*} y} \\
& E_{R 1}=\frac{\left(y^{T} H y\right)^{2} E_{H}-\left(y^{T} \sigma\right)^{2} E_{H}{ }^{*}}{\left(y^{T} H y\right)^{2}-\left(y^{T} \sigma\right)^{2}}=\frac{\left(y^{T} H y\right)^{2} E_{H}-\left(y^{T} H^{*} y\right)^{2} E_{H^{*}}}{\left(y^{T} H y\right)^{2}-\left(y^{T} H^{*} y\right)^{2}} \\
& E_{H}=\gamma E_{R 2}+(1-\gamma) E_{R 1} \\
& E_{H^{*}}=1 / \gamma E_{R 2}+(1-1 / \gamma) E_{R 1}
\end{aligned}
$$

$$
\gamma=\left(\frac{y^{T_{\sigma}}}{y^{T} H y}\right)
$$

It is especially interesting that the two variationally derived correction terms $E_{H}$ and $E_{H^{*}}$ give rise to a one-parameter family of correction terms $E=\alpha E_{H}+$ $(1-\alpha) E_{H^{*}}$ whose corresponding variable-metric methods are "exact." The DFP-rank-2 and rank-1 correction terms are members of this one-parameter family that correspond to particularly interesting choices for the parameter $\alpha$. This family includes all symmetric variable-metric correction terms that have been published [1], [2], [3], [4], [6], [7].**

In fact, it is equivalent to the one-parameter family given by Broyden's algorithm 2 [1]. The equivalence can be obtained by setting

$$
\alpha=\frac{\left(1-\beta y^{T} \sigma\right) y^{T} H y}{y^{T} H y+y^{T} \sigma},
$$

where $\beta$ is Broyden's parameter.

Broyden's algorithm 1 (i.e., the rank-1 algorithm) is just a special case of his algorithm 2 [1], with $\beta=1 /\left(y^{T} H y-y^{T} \sigma\right)$; a point that seems to have been overlooked by Broyden himself.

It is also possible to obtain $E_{R 1}$ and $E_{R 2}$ directly from Eq. (3) by choice of a suitable $M$. For the rank-1 case a choice that works is

$$
M_{R 1}=H^{*}-H=E \text {. }
$$

However, using $M_{R 1}=M$ in Eq. (3) yields $E=E_{R 1}$ which has rank 1 and, hence, $M_{R 1}$ has no inverse.

** Davidon's variance algorithm [3] multiplies the rank-1 correction term $E_{R 1}$ by a scalar function of $\left(g^{T} \mathrm{Hg}^{*} / \mathrm{g}^{* T} \mathrm{Hg}^{*}\right)$ so as to ensure the stability of the method. 
Before going further, we note that:

(i) Formula (3) is homogeneous in $M$; therefore, replacing $M$ by $\mu M$, where $\mu$ is a scalar, has no affect on the resultant $E$.

(ii) $M$ always appears in conjunction with $y$ in formula (3) either as $M y$ or as $y^{T} M$; therefore, the replacement of $\left(y^{T} H y\right) H$ by $H y y^{T} H$ and $\left(y^{T} \sigma\right) H^{*}=\left(y^{T} H^{*} y\right) H^{*}$ by $H^{*} y y^{T} H^{*}$ as terms of $M$ has no affect on the resultant $E$.

Hence the substitution of either

$$
M_{R 1}=H^{*}-\frac{H y y^{T} H}{y^{T} H y}
$$

or

$$
M_{R 1}=H-\frac{\sigma \sigma^{T}}{\sigma^{T} y}
$$

for $M$ in Eq. (3) also yields $E_{R 1}$.

Substitution of any of the forms of $M_{R 2}$ given below in Eq. (3) is sufficient to show that all give rise to the DFP correction term $E_{R 2}$.

$$
\begin{aligned}
& M_{R 2}=\left(y^{T} H y\right)^{1 / 2} H^{*}-\left(y^{T} \sigma\right)^{1 / 2} H, \\
& M_{R 2}=\left(y^{T} H^{*} y\right)^{-1 / 2} H^{*}-\left(y^{T} H y\right)^{-1 / 2} H, \\
& M_{R 2}=H^{*}-\left(\frac{y^{T} \sigma}{y^{T} H y}\right)^{1 / 2} \frac{H y y^{T} H}{y^{T} H y}, \\
& M_{R 2}=H-\left(\frac{y^{T} H y}{y^{T} \sigma}\right)^{1 / 2} \frac{\sigma \sigma^{T}}{y^{T} \sigma} .
\end{aligned}
$$

Although the matrices $M_{R 1}$ and $M_{R 2}$ given by expressions (7) through (9) are, in general, nonsingular, these choices for $M$ and hence, the corresponding $W$ 's are not necessarily positive-definite. Thus, their substitution in Eq. (3) is somewhat contrived. Just what role they play in the variational derivation of the rank-1 and DFP rank-2 methods remains confusing.

City College of the City University of New York New York, New York 10031

1. C. G. Broyden, "Quasi-Newton methods and their application to function minimisation," Math. Comp., v. 21, 1967, pp. 368-381. MR 36 \#7317.

2. W. C. Davidon, Variable Metric Method for Minimization, A. E. C. Res. and Develop. Report ANL-5990 (Rev. TID-4500, 14th ed.) 1959.

3. W. C. DAvidon, "Variance algorithm for minimization," Comput. J., v. 10, 1968, pp. 406410. MR $36 \# 4790$.

4. R. Fletcher \& M. J. D. Powell, "A rapidly convergent descent method for minimization," Comput. J., v. 6, 1963, pp. 163-168. MR 27 \#2096.

5. D. GoldFARB, "Sufficient conditions for the convergence of a variable metric algorithm," Proc. Confer. on Optimization (University of Keele, 1968). (To appear.)

6. J. GrennstadT, "Variations on variable metric methods," Math. Comp., v. 24, 1970, pp. 1-22.

7. P. Wolfe, Another Variable Metric Method, Working Paper, 1967. 